\title{
Development of control system for optimal stress during cardiac MR spectroscopy
}

\author{
Hee-Won Kim ${ }^{*}$, Karam Souibri ${ }^{1}$, Don Lee $^{2}$ and Gerald Pohost ${ }^{1}$
}

\author{
Address: ${ }^{1}$ University of Southern California, Los Angeles, CA, USA and ${ }^{2}$ Hollywood Heart and Vascular Institute, Los Angeles, CA, USA \\ * Corresponding author
}

from 13th Annual SCMR Scientific Sessions

Phoenix, AZ, USA. $21-24$ January 2010

Published: 21 January 2010

Journal of Cardiovascular Magnetic Resonance 20 I0, I2(SuppI I):PI2I doi:I0.I I86/I532-429X-I2-SI-PI2I

This abstract is available from: http://jcmr-online.com/content/I2/SI/PI2I

(C) 2010 Kim et al; licensee BioMed Central Ltd.

\section{Introduction}

To induce changes in myocardial metabolism monitored by ${ }^{31} \mathrm{P}$ MRS in patients with symptoms of coronary artery disease, handgrip stress test is frequently used. At a level of stress of $30 \%$ maximum voluntary contraction, it is critical to maintain a continuous stress level. Otherwise, the phosphate metabolic alterations tend to recover quickly, and any changes would disappear with involuntary loss of the stress. In practice, it is very difficult for patients to maintain a constant pressure level and involuntary adaptation of the patient would interfere with the measurement of such changes.

\section{Purpose}

The purpose of this work is to develop a PC-based handgrip stress control system in order to improve precision of phosphate metabolic alterations during the cardiac stress P-31 MRS. The system is for the use within the high field clinical MR scanner.

\section{Methods}

A compression-load-cell handgrip was chosen to minimize potential inductive coupling noise. The load-cell/ strain-gauge provides the signal source and the digital signal from the handgrip is primarily sent to the PC serial port through an RS-232 data interface. The signal was received using HyperTerminal and Matlab ${ }^{\oplus}$ serial port. The control band was set such that an error signal would be induced when the stress level is out of the set band. By the feed-forward or feedback control, the error-signals actuate audio-visual stimulation to the subject in the $3 \mathrm{~T}$ magnet.

\section{Results}

The automatic control system successfully provided feedback to human subjects to maintain a continuous and constant stress level during the stress session. Two different time courses of the stress from the same subject are illustrated (Figure). It is shown that the stress level was far more stable during the entire session for the case with automatic control system than that with verbal communication. The ${ }^{31} \mathrm{P}$ MR spectra were obtained from four healthy volunteers. The PCr/ATP changes during the stress was not significantly different between acquisitions with and without the control system ( $\mathrm{p}>0.15)$. The spectra obtained with the control system showed better spectral resolution ( $<4 \mathrm{~Hz}$ FWHM of PCr peak).

\section{Conclusion}

It was demonstrated that the automated stress control system has made the stress level more constant in the MR scanner. In light of this improvement, one can obtain more accurate measurements of the changes in metabolites, and higher spectral resolution by stabilizing movements with proactively sustained control during stress ${ }^{31} \mathrm{P}$ MRS.

\section{Acknowledgements}

This study was supported by DOE DE-FG02-06ER64322. 